\title{
Semiconductor Nanorod Liquid Crystals
}

\author{
Liang-shi Li, Joost Walda, Liberato Manna and A. Paul Alivisatos* \\ Department of Chemistry \\ University of California, Berkeley \\ Berkeley, CA 94720 \\ Materials Science Division \\ Lawrence Berkeley National Laboratory \\ 1 Cyclotron Road \\ Berkeley, CA 94720
}

* To whom correspondence should be addressed. Email: alivis@uclink4.berkeley.edu.

\begin{abstract}
Rodlike molecules form liquid crystalline phases with orientational order and positional disorder. The great majority of materials in which liquid crystalline phases have been observed are comprised of organic molecules or polymers, even though there has been continuing and growing interest in inorganic liquid crystals. Recent advances in the control of the sizes and shapes of inorganic nanocrystals allow for the formation of a broad class of new inorganic liquid crystals. Here we show the formation of liquid crystalline phases of CdSe semiconductor nanorods. These new liquid crystalline phases may have great importance for both application and fundamental study.
\end{abstract}


Inorganic nanocrystals may be viewed as an emerging new class of macromolecules. Since methods were first developed to encapsulate nanocrystals with a monolayer of protective surfactant $(1,2)$, nanocrystals have been assembled as active components inside plastic electronics $(3,4,5)$, and they have been assembled into dimers, trimers $(6)$, and crystals of nanocrystals $(7,8)$. In each case the nanocrystals are treated as a conventional polymer or biological macromolecule from the assembly point of view. This enables a wide range of chemical macromolecular assembly techniques to be extended to inorganic solids, which possess a diverse range of optical, electrical, and magnetic properties (9). One important characteristic of organic macromolecules is their propensity to form liquid crystalline phases when they possess a rod-like shape $(10,11)$. Over the last several decades there has been continuing and growing interest in inorganic liquid crystals, but the studies have been limited by available samples to molecular wires or ribbons, or highly polydisperse insulating colloidal disks and rods such as gibbsite $\left(\mathrm{Al}(\mathrm{OH})_{3}\right)$ and boehmite $(\mathrm{AlO}(\mathrm{OH}))(12,13)$. The poor monodispersity of these colloidal rods also prevents the quantitative comparison between experiments and theories (14). The advent of new methods for preparing highly soluble and processable colloidal metallic, semiconductor, and magnetic nanocrystals (15-20) with very tight control over size and shape creates significant new opportunities in the study of inorganic liquid crystals. In this paper we demonstrate that semiconductor colloidal nanorods spontaneously form liquid crystalline phases in concentrated solution, and we discuss the potential implication of these new phases both for the theory of liquid crystal formation and for the practical application of liquid crystals. 
In this work we have focused on nanorods of CdSe, because they can be synthesized in large quantity $(\sim 100 \mathrm{mg})$ with variable aspect ratio and excellent monodispersity (17, 21). The CdSe nanorods are made by pyrolysis of organometallic precursors of $\mathrm{Cd}$ and $\mathrm{Se}$ in a hot surfactant mixture. By varying the composition of the surfactant we synthesize rod-like CdSe nanocrystals with variable width and length (21). Typically the aspect ratio can be varied from 1 to 15 , width from 3 to $7 \mathrm{~nm}$, and length from 3 to $70 \mathrm{~nm}$, while the distribution is typically $5 \%$ for width and $10 \%$ for length. The optical properties of the nanorods depend strongly upon diameter and length due to quantum size effects (22). The photoluminescence wavelength can be tuned over the visible range by variation of the particle size, and the degree of polarization can be controlled by variation of the aspect ratio (21). The transmission electron micrographs of several such samples shown in figure 1 illustrate the range and quality of the nanorods produced by this method. These CdSe nanorods are functionalized with amphiphilic molecules, such that a polar functional group is bound to the nanocrystal surface, and long alkyl chains are projecting outward. These alkyl chains provide high solubility of the nanorods in organic solvent.

The formation of liquid crystalline phases of the $\mathrm{CdSe}$ nanorods was verified by examining birefringence in solutions of nanorods in an optical microscope with crossed polarizers. Even quick drying of the solution of CdSe nanorods with aspect ratio of 10 on a glass slide can make a birefringent pattern, indicating local orientational order. In order to study the evolution of the birefringence, we seal a solution of CdSe nanorods (in cyclohexane, $10 \%$ wt.) with a length of $40.0 \mathrm{~nm}$ and a width of $4.0 \mathrm{~nm}$ in a capillary tube. A $100 \mathrm{~W}$ halogen lamp is used as the light source. The solution itself is isotropic at such a low nanorod concentration. When it is mounted on the microscope, the heating caused 
by the incident light slowly evaporates the solvent at the focusing spot, causing the solution to become more concentrated, leading to the formation of a birefringent liquid crystalline phase. The anisotropic refractive index of the liquid crystalline phase alters the polarization of transmitted light, leading to light and dark patterns on the micron scale. Because the capillary tube is sealed and the light only changes the distribution of solvent within the tube, we can change the local concentration of the solution by varying the intensity of incident light, and we can also control the speed of evaporation and monitor the evolution of the liquid crystalline phase reversibly. Figure 2 shows the birefringent pattern observed at two different stages. Isotropic liquids show complete extinction, and no pattern is observed from concentrated solutions of mixtures of the two surfactants used in the synthesis of these nanorods (trioctylphosphine oxide and tetradecylphosphonic acid). The intensity of the transmitted light reveals the orientation of the nanorods.

Figure 2(A) shows the nucleation of the liquid crystalline phase. Small $(\sim 2 \mu \mathrm{m})$ and large $(\sim 10-20 \mu \mathrm{m})$ birefringent droplets (tactoids) emerge from an isotropic solution as the concentration increases. The solution appears red because of the band gap of the CdSe nanocrystals. As they have higher optical density than the isotropic medium we conclude that these droplets have higher concentration of nanorods. The variations in the intensity of the transmitted light through the droplets indicate alignment of the rods within each droplet. The patterns are generally dipolar, but some higher modes have been observed as well. The slightly elongated non-spherical shape of the droplets provides another clear indication that the nanorods are oriented within each droplet. This distortion is due to the balance between surface tension and anisotropic elasticity (23), in 
which the former favors a spherical surface while the latter favors a cylindrical uniaxial surface. This effect is more obvious in smaller droplets than in bigger ones.

Once the liquid crystalline phases have fully developed, a new set of patterns is observed. Figure 2(B) shows the Schlieren structures formed at a later stage. Dark threadlike brushes are observed sticking out from some singular points. The singular points (disclinations) have been identified as the positions where the alignment direction of particles is not well defined. The dark brushes are due to the particles with alignment parallel to either of the polarizers. When the sample is rotated, these singular points remain fixed but the dark brushes move around them. When one end of the capillary tube is lifted the patterns flow and deform easily, suggesting the fluidity of the birefringent phase. Similar observations were also reported in colloidal boehmite rod suspensions (24).

To demonstrate it is possible to form a stable liquid crystalline phase in $\mathrm{CdSe}$ nanorod solutions, we dissolve the nanorods with a diameter of $3.8 \mathrm{~nm}$ and length of $49.0 \mathrm{~nm}$ in excess of anhydrous cyclohexane and then blow some solvent away with nitrogen. Figure 3 shows some images taken under a polarizing optical microscope from a solution with CdSe nanorods $\sim 88 \%$ by weight ( 50\% v./v.), with 3(A) showing the solution as a mixture of a liquid crystalline droplets and an isotropic phase one day after homogenization. Although a complete phase separation may take months, two weeks later large domains of liquid crystalline phase can be seen by eye. Figure 3(B) shows an image of the interface between the liquid crystalline phase and the isotropic phase obtained after the droplets have grown and coalesced. Schlieren structure can also be seen. When the solution is diluted to $\sim 80 \%$ ( 38\% v./v.), the liquid crystalline domains 
disappear, and the solution becomes isotropic. Shaking the solution will cause flow birefringence, which slowly vanishes when at rest. This concentration is significantly higher than the critical concentration expected from numerical study (25) performed on hard spherocylinders, even when the change of effective volume caused by the surface capping ligands is considered (26). The shape of the intermolecular potential between the nanorods will influence the predicted phase diagram. A careful study of the phase diagram of the nanorod liquid crystalline phases is underway to make a detailed comparison to theory.

Figures 3(C) and 3(D) show some defects observed with Schlieren structures characteristic of a nematic phase. 3(C) shows disclinations with strength of $1 / 2$ Strength of a disclination is defined to be a quarter of the number of dark brushes sticking out of it. In 3(D) we see disclinations with strength of 1 . Interestingly, the disclinations with strength of 1 are observed much less often than those with strength of $1 / 2$ and sometimes even slight heating by light can anneal them out. Because the formation of disclinations in liquid crystalline phases costs elastic energy (27), and the energy involved increases with strength, we can conclude that the liquid crystalline phase of CdSe nanorods have much higher elastic constants compared to regular organic liquid crystal molecules, as expected from the high rigidity and the large size of the CdSe nanorods.

One interesting feature of inorganic liquid crystals is the high electron density and electron scattering power of the atoms as compared to carbon. This potentially enables transmission electron microscopy (TEM) as a means of directly visualizing the local order, provided the liquid can be "frozen" on a substrate without altering the order. A combination of electron microscopy and optical microscopy has been used to investigate 
the formation of tactoid structures in $\mathrm{K}_{2} \mathrm{O}_{5}$ sol (28). By TEM we have observed a wide variety of remarkable liquid-crystalline type self-assemblies from nanorod multilayers, but it remains to be proven whether these patterns truly represent the degree of order in the liquid crystalline phase. A freshly cleaved mica sheet coated with a thin amorphous carbon film is immersed vertically into a dilute solution. As the solvent evaporates, the liquid level sweeps over the substrate, depositing a multilayer of CdSe nanorods behind when the solvent is completely gone. The carbon film is then floated off on water and picked up on a TEM grid.

Such experiments have been performed on $4.0 \times 40.0 \mathrm{~nm}$ and $3.7 \times 18.0 \mathrm{~nm}$ (Figure 4) nanorods of CdSe. We have been able to image both "smectic-A" and "nematic" types of order in multilayer films of both nanorod samples, as well as crystalline superlattices (29). Quick drying of droplets of CdSe nanorod solution on glass slides under a polarizing optical microscope shows formation of Schlieren structures at the edges of the droplets where the optical density is also the highest. This strongly suggests that the multilayer TEM patterns observed here have formed in the liquid prior to complete solvent evaporation. This can be explained by the convective transfer of CdSe nanocrystals from the bulk of the solution $\mathfrak{b}$ the edges caused by the solvent evaporation (30), which makes the local concentration high enough for the isotropic-liquid crystalline phase transition. Until now we have not observed smectic phases in concentrated solution; however, smectic phases have been predicted by Monte Carlo simulation (31) and have been observed in a variety of systems $(12,13)$. Formation of smectic phases requires a solution of higher concentration, which is not easily achievable in our system. Freeze-fracture experiments have to be performed to further test whether these patterns 
can be assigned to the liquid crystalline phases of the nanorods, or if the patterns somehow arise as a solid-liquid equilibrium during the evaporation, as reported in solid superlattices of $\mathrm{Au}$ nanorods (32). In any case, these oriented multilayer assemblies of the nanorods are in themselves rather interesting objects for future studies of anisotropic 3-dimensional artificial quantum solids.

The anisotropy of intermolecular interactions plays an important role in the formation of liquid crystals. Because CdSe nanorods can be synthesized with variable aspect ratio and excellent monodispersity, and they can be dispersed at high density in organic solvent, we believe they may prove to be an excellent model system in the study of liquid crystalline phases to compare with theories, especially for systems consisting of short rods. On the other hand, the orientation of liquid crystals is very sensitive to external field and pretreated surface (27), and therefore liquid crystalline phases of CdSe nanorods provide a way to align and manipulate their orientation on a large scale. Because they have tunable and linearly polarized photoluminescence as well as anisotropic nonlinear optical properties (33), liquid crystals of CdSe nanorod can potentially be used as functional components in electro-optical devices such as polarized light emitting diodes, flat panel displays and electro-optic modulators. 


\section{Caption for figures}

Fig. 1 Transmission electron micrograph of CdSe nanorods. (A) - (C) Nanocrystals with width of ca. $3.2 \mathrm{~nm}$ and length of 11, 20, and $40 \mathrm{~nm}$ respectively. (D) - (F) Nanocrystals with width of $c a .4 .2 \mathrm{~nm}$ and length of 11,20 , and $40 \mathrm{~nm}$, respectively.

Fig. 2 Birefringence observed under a polarizing optical microscope during evaporation of solvent. (A) Droplets (tactoids) formed from the isotropic phase with the evaporation of solvent. (B) Schlieren textures in a later stage. The red background is due to the absorption of CdSe nanocrystals. The scale bars are $20 \mu \mathrm{m}$.

Fig. 3 Images of liquid crystalline phase in concentrated solution of CdSe nanocrystals under a polarizing optical microscope. Isotropic phase appears dark in these images. All the bright areas in the image correspond to the liquid crystalline phase, with dark lines corresponding to disclinations or the area where the orientation of the nanorods are parallel to either of the polarizers. (A) Droplets of liquid crystalline phase mixed with isotropic phase. (B) Interface between a big liquid crystalline droplet (right) and isotropic phase (left). (C) and (D) Disclinations of strength of 1/2 (C) and 1 (D) respectively. The arrows point to the disclinations.

Fig. 4 TEM images of the structures of $4.0 \times 40.0 \mathrm{~nm}(\mathrm{~A}$ and $\mathrm{B})$ and $3.7 \times 18.0 \mathrm{~nm}(\mathrm{C}$ and D) CdSe quantum rods formed by solvent evaporation. (A) and (C) show nematic order, and the layered structure in (B) and (D) suggests a smectic-A type order. The scale bars are $50 \mathrm{~nm}$. 


\section{Reference}

1. M. L. Steigerwald, et al., J. Am. Chem. Soc. 110, 3046 (1988).

2. G. Schmid, L. F. Chi, Adv. Mat. 10, 515 (1998).

3. V. Colvin, M. Schlamp, A. P. Alivisatos, Nature 370, 354 (1994).

4. B. O. Dabbousi, M. G. Bawendi, O. Onotsuka, M. F. Rubner, Appl. Phys. Lett. 66, 316 (1998).

5. W.U. Huynh, X. Peng, A.P. Alivisatos, Adv. Mat. 11, 923 (1999).

6. C. J. Loweth, W.B. Caldwell, X. Peng, A.P. Alivisatos, P.G. Schultz, Angew. Chem., Int. Ed. Engl. 38, 1808 (1999) .

7. C. B. Murray, C. R. Kagan, M. G. Bawendi, Science 270, 1335 (1995).

8. C. A. Mirkin, R. L. Letsinger, R. C. Mucic, J. J. Storhoff, Nature 382, 607 (1996).

9. A. P. Alivisatos, Science 271, 933 (1996).

10. J. C. Horton, A. M. Donald, A. Hill, Nature 346, 44 (1990).

11. T. E. Strzelecka, M. W. Davidson, R. L. Rill, Nature 331, 457 (1988).

12. J.-C. P. Gabriel, P. Davidson, Adv. Mat. 12, 9 (2000).

13. A. S. Sonin, J. Mat. Chem. 8, 2557 (1998).

14. G. J. Vroege, H. N. W. Lekkerkerker, Rep. Prog. Phys. 55,1241 (1992).

15. M. Li, H. Schnablegger, S. Mann, Nature 402, 393 (1999).

16. T. S. Ahmadi, Z. L. Wang, T. C. Green, A. Henglein, M. A. El-Sayed, Science 272, 1924 (1996).

17. X. Peng, et al., Nature 404, 59 (2000).

18. H. J. Dai, et al. Nature 375, 769 (1995).

19. S. Chang, C. Shih, C. Chen, W. Lai, C. R. C. Wang, Langmuir 15, 701 (1999).

20. V. F. Puntes, K. M. Krishnan, A. P. Alivisatos, Science 291, 2115 (2001).

21. J. Hu, L.-S. Li, W. Yang, L. Manna, L.-W. Wang, A. P. Alivisatos, Science 292, 2060 (2001).

22. L.-S. Li, J. Hu, W. Yang, A. P. Alivistaos, Nano Lett. 1, 349 (2001).

23. J. D. Bernal, I. Frankuchen, J. Gen. Physiol. 25, 111 (1941).

24. P. A. Buining, H. N. W. Lekkerkerker, J. Phys. Chem. 97, 11510 (1993).

25. S-D. Lee, J. Chem. Phys. 87, 4972 (1987). 
26. Assume the surface molecules increase both diameter and length by $1.1 \mathrm{~nm}$ (trioctylphosphine oxide). A critical concentration of $27 \% \mathrm{v} . / \mathrm{v}$. is given for hard rods with an aspect ratio of 10 in ref. 25.

27. S. Chandrasekhar, Liquid Crystals (the University Press, Cambridge, 1992).

28. J. H. L. Watson, W. Heller, W. Wojtowicz, Science 109, 274 (1949).

29. L.-S. Li, et al, unpublished result.

30. N. D. Denkov, et al, Nature 361, 26, 1993.

31. D. Frenkel, H. N. W. Lekkerkerker, A. Stroobants, Nature 332, 822 (1988).

32. B. Nikoobakht, Z. L. Wang, M. A. El-Sayed, J. Phys. Chem. B 101, 8635 (2000).

33. L. Brus, Appl. Phys. A 53, 465 (1991).

34. We thank F. Kim and S.-C. Hong for the use of the polarizing microscope, and the Electron Microscope Lab at UC Berkeley for TEM. This work is supported by the Director, Office of Energy Research, Office of Science, Division of Materials Sciences, of the U.S. Department of Energy under Contract No. DE-AC03-76SF00098 and by the Air Force Office of Scientific Research (F49620-98-1-0243). J. W. thanks FOM-Institute for Atomic and Molecular Physics, Amsterdam, the Netherlands for a scholarship. 


\section{Figure 1}
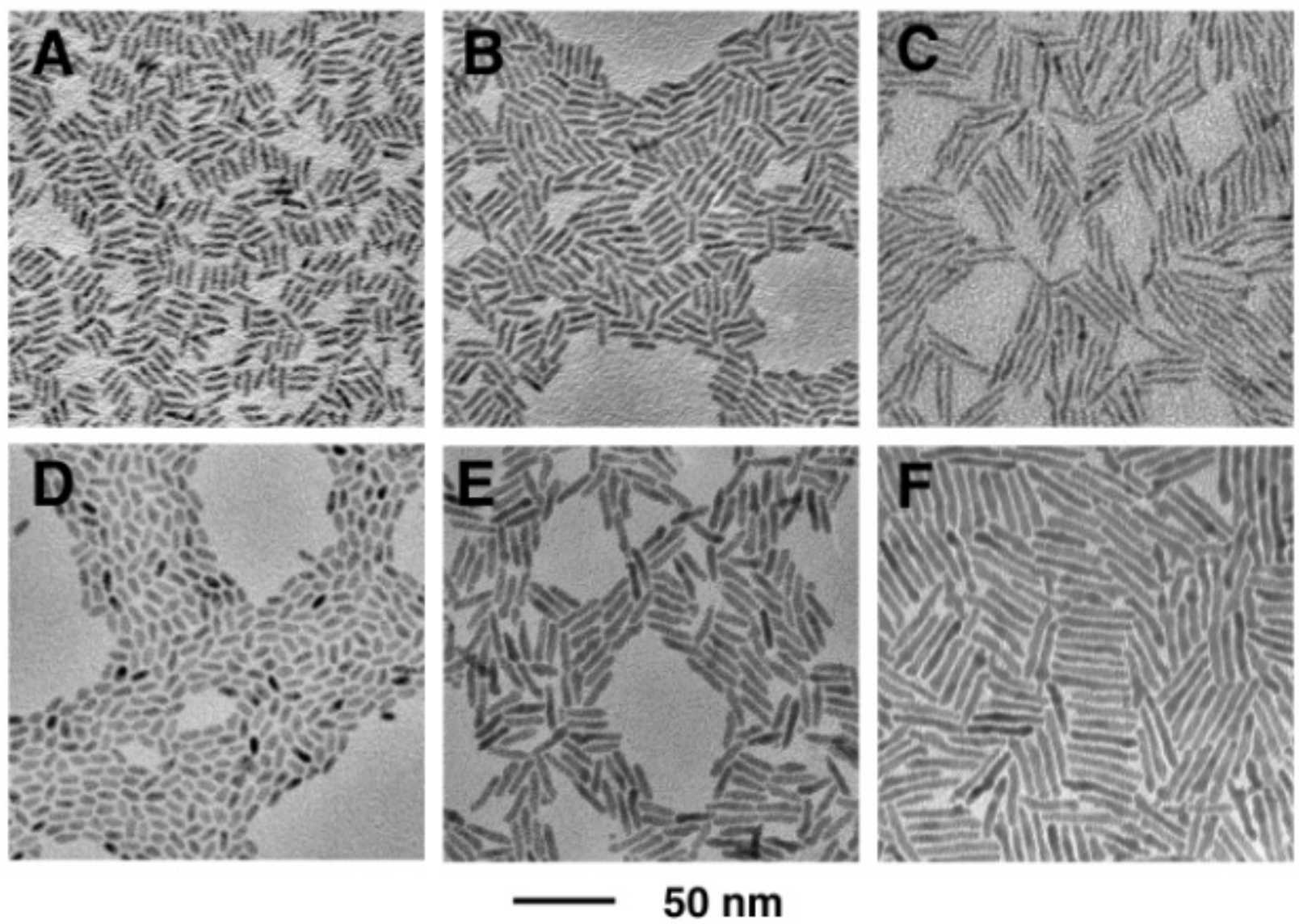
Figure 2

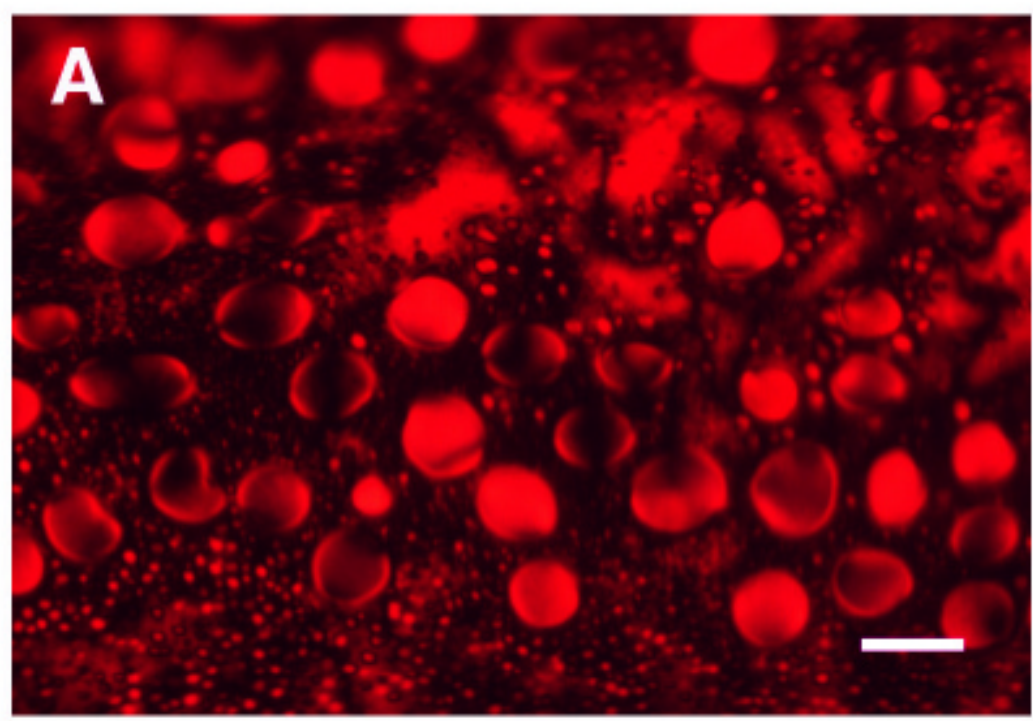

B 
Figure 3
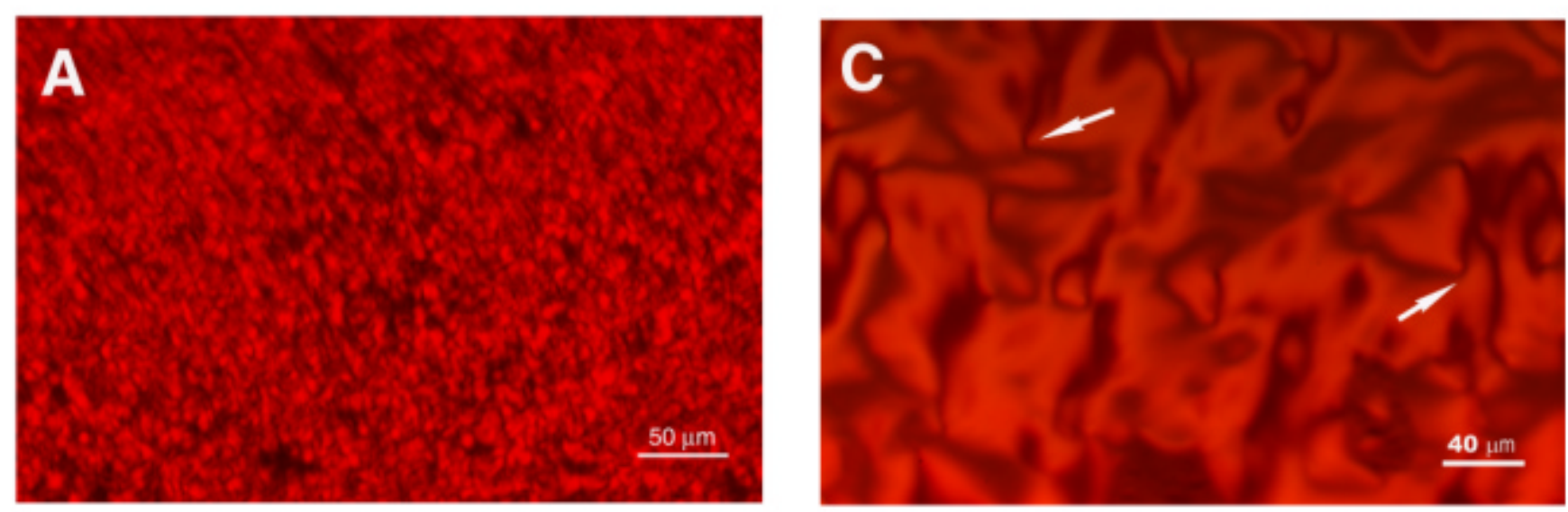

\section{B}

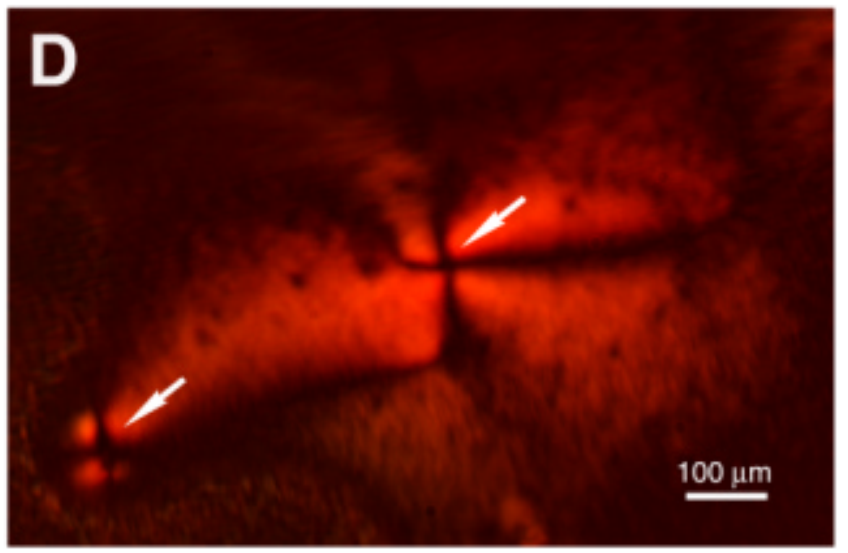


Figure 4
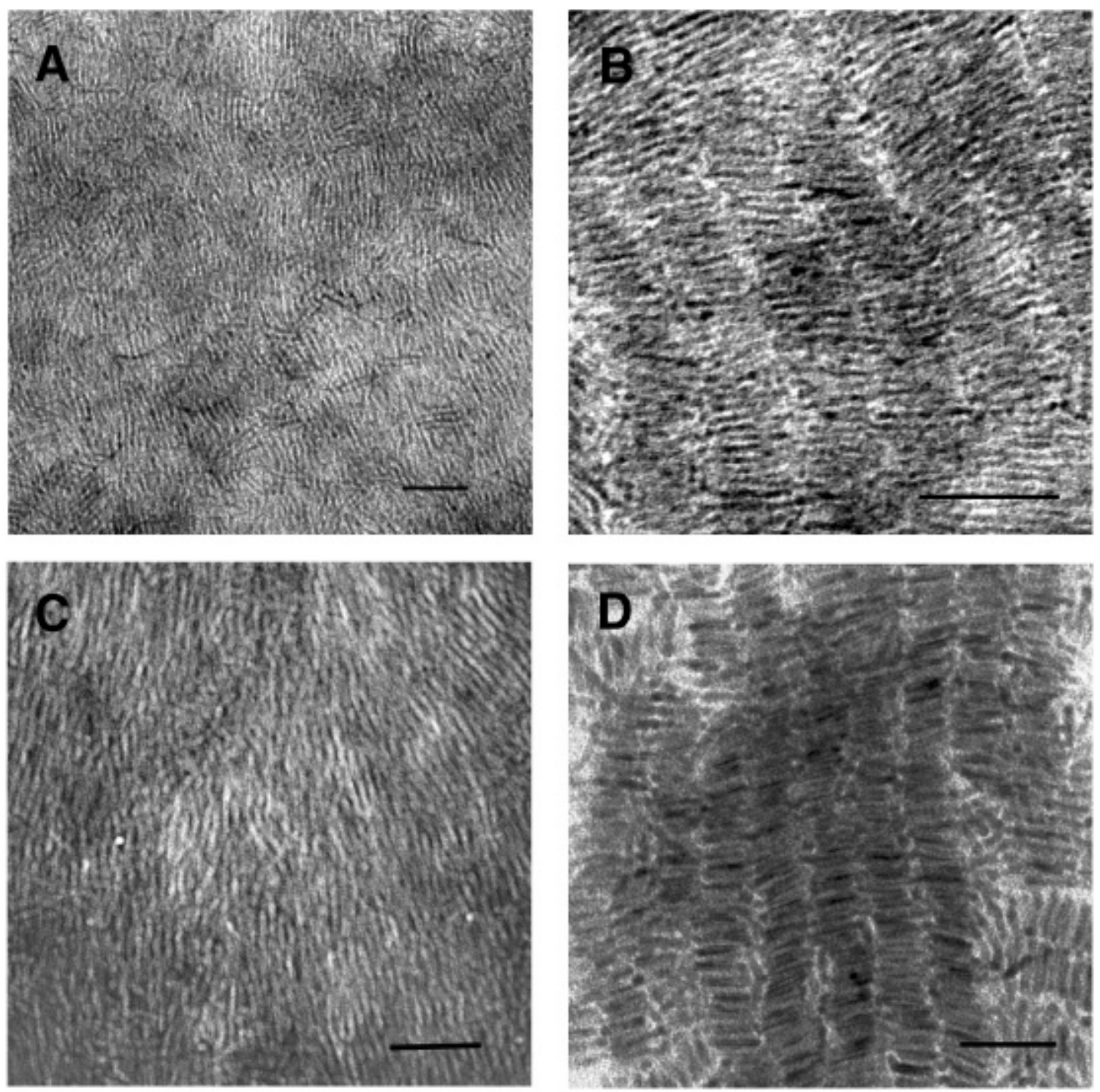